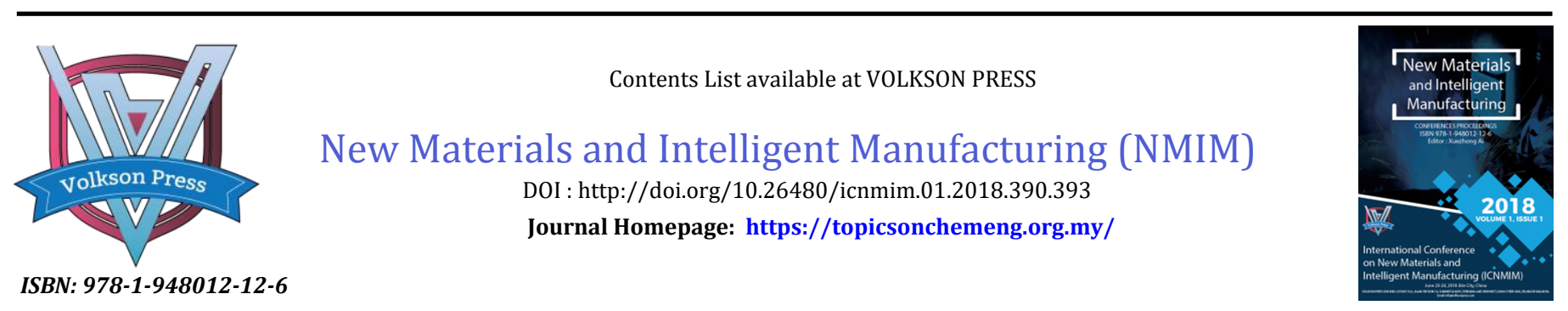

\title{
RECOGNITION METHOD OF POWER QUALITY DISTURBANCE SIGNALS BASED ON IMAGE FEATURES
}

\author{
Lin Lin ${ }^{1 *}$, Liu Zuoming ${ }^{2}$ \\ ${ }^{1}$ College of Information and Control Engineering, Jilin Institute of Chemical Technology, Jilin 132022, China. \\ ${ }^{2}$ State Grid Jilin Electric Power Research Institute, Changchun 130021, China.
}

This is an open access article distributed under the Creative Commons Attribution License, which permits unrestricted use, distribution, and reproduction in any medium, provided the original work is properly cited.

\section{ARTICLE DETAILS}

Article History:

Received 26 June 2018

Accepted 2 July 2018

Available online 1 August 2018

\section{ABSTRACT}

In order to meet the requirements of power quality monitoring and grid active control of distributed generation, in this paper, a new method to identify power quality transient disturbances by digital image processing is proposed. First, the power quality signal in the form of one-dimension time series was converted into two-dimensional digital image, and then the image feature enhancement was carried out by Gamma change. A unified feature extraction method of disturbance signal was established, and the automatic recognition method of disturbance type was established to improve the feature extraction efficiency. The results indicated that the proposed method performed a high efficiency and low hardware cost than existing methods in power quality signal recognition, which can further improve the power quality and reliability of distribution network.

\section{KEYWORDS}

Power quality, digital image, Gamma transform, feature extraction.

\section{INTRODUCTION}

Distributed generation, represented by wind and solar energy, has been connected to the distribution network on a large scale. As a result, new power quality problems have been brought to the power grid. At the same time, along with the enhancement of the intellectualization of power grid, the wide application of power electronic equipment in the power grid further worsens the power quality problems such as harmonics and oscillations. At present, there are detailed standards for the power quality provided by distributed generation both at home and abroad [1]. In order to ensure the stability and reliability of the power system, power grid management enterprises need to add a large number of power quality signal acquisition and analysis equipment, which can address and analyze the responsibility of the disturbance source in the power grid. And on this basis, to carry out targeted governance.

Because of the complex power quality analysis process and the high cost of hardware and communication, the cost of power quality monitoring and analysis of distribution network become higher, and the related work is seriously lagging behind [2]. Therefore, it is an efficient, simple and easy to realize power quality transient disturbance analysis method, and then use this method to reduce the cost of hardware. It is the key point to solve the technology of power quality monitoring and analysis.

Power quality disturbance signal recognition method is mainly divided into 2 parts: feature extraction and pattern recognition [3]. Feature extraction is based on signal processing, and then extract time-frequency features [4]. The traditional methods of signal processing mainly include time-frequency analysis by using fast Fourier transform and short time Fourier transform. The analytical ability of these methods is limited, and the number of disturbances is less. Wavelet transform and S transform show many advantages such as large amount of computation, high hardware requirements and low real-time performance [5]. of power quality signal to gray image, then depicts the disturbance characteristics by different image enhancement techniques, and achieves a certain effect [6]. However, this study used different image processing methods for different types of disturbance signals, but how to determine which image processing methods and what characteristics to adopt was not discussed. The method is not automated and can not be applied to industrial site. However, it has made a useful exploration for the application of image processing methods in the field of disturbance recognition.

Pattern recognition commonly used methods include neural network, support vector machine, extreme learning machine and so on. Artificial neural network (ANN) is widely used in practical applications because of its simple structure, adjustable parameters, many training algorithms and good maneuverability. In this paper, firstly, the power quality signal simulation modeling is carried out. Then, the one-dimensional power quality disturbance signal is transformed into a two-dimensional gray level image, and the image enhancement technique was used to deal with the gray image of various types of disturbance signals, and the disturbance features in the two-dimensional image were further highlighted. On this basis, the disturbance characteristics were extracted respectively. Finally, on the basis of a large number of simulation and measured signal analysis, the BP neural network was used for modeling, and the automatic recognition of the disturbance signals was realized by using the established model.

\section{SIMULATION MODELING OF POWER QUALITY SIGNAL}

This paper mainly classifies the Sag, Swell, harmonic, interruption and transient power quality signal. The simulation models of various power quality disturbances are as follows [5]: Standard signal:

$$
v(t)=A \cos (\omega t) \quad A=1(p u), f=50 H z, \omega=2 \pi f ; u(t)=\left\{\begin{array}{l}
1 t \geq \\
0 t \leq
\end{array}\right.
$$


Sag:

$v(t)=A\left\{1-k\left[u\left(t_{2}\right)-u\left(t_{1}\right)\right]\right\} \cos (\omega t)$

$0.1<k<0.9 ; T \leq t_{2}-t_{1} \leq 9 T$

Swell:

$v(t)=A\left\{1+k\left[u\left(t_{2}\right)-u\left(t_{1}\right)\right]\right\} \cos (\omega t)$

$0.1<k<0.9 ; T \leq t_{2}-t_{1} \leq 9 T$

Harmonics:

$v(t)=A \cos (\omega t) \quad h_{2} \cos (2 \omega t)+h_{3} \cos (3 \omega t)+h_{5} \cos (5 \omega t)+h_{7} \cos (7 \omega t)+h_{9} \cos (9 \omega t)+h_{11} \cos (11 \omega t)$ $0 \leq h<0.25, i=3,5,7 ; 0 \leq h_{i}<0.1, i=2,9,11$;

Interruption:

$v(t)=A\left\{1+k\left[u\left(t_{2}\right)-u\left(t_{1}\right)\right]\right\} \cos (\omega t)$ $0.9 \leq k<1 ; T \leq t_{2}-t_{1} \leq 9 T$

Transient:
$v(t)=A\left\{\cos (\omega t)+k \exp \left[-\left(t-t_{1}\right) / \tau\right] \cdot \cos \left[\omega_{n}\left(t-t_{1}\right)\right] \cdot\left[u\left(t_{2}\right)-u\left(t_{1}\right)\right]\right\} \cos (\omega t)$

$$
k=0.7 ; \tau=0.015 ; \omega=2 \pi f ; 900 \mathrm{~Hz} \leq f_{n} \leq 1300 \mathrm{~Hz}
$$

According to the above model, the simulation signal is generated by Matlab, the fundamental frequency of the signal is $50 \mathrm{~Hz}$, and the sampling rate is $3.2 \mathrm{kHz}$.

\section{IMAGE ENHANCEMENT TECHNOLOGY}

Image enhancement technology is mainly used to highlight the disturbance components of the original signal, and facilitate the extraction of feature and disturbance type recognition in the later stage [6].

For grayscale images, the range of pixel values is $0-255$, of which 0 represents black and 255 represents white. The waveform of the original analog signal is sampled, and each sampling point after the sampling corresponds to a pixel of the gray image. By normalization, the range of sampling points is $0-255$. The sine wave is transformed into a grayscale image, as shown in the following figure 1 :

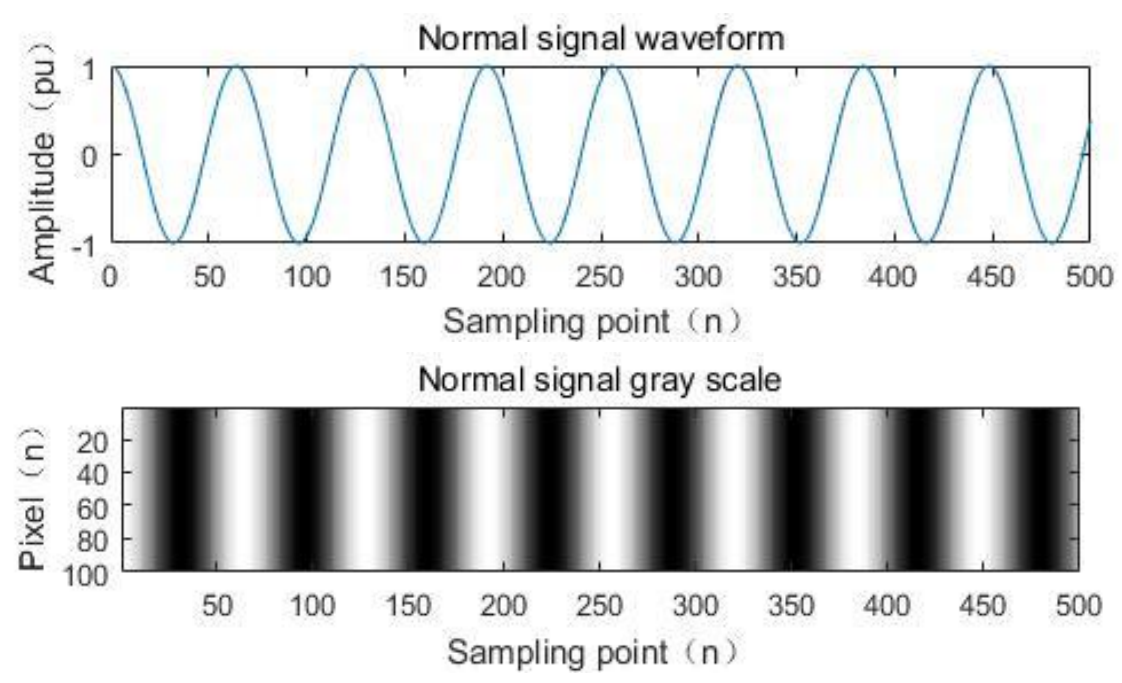

Figure 1: Crayscale Transformation Effect Diagram

Figure1 shows that after transforming the standard power quality signal into a grayscale image, the gray scale transformation is uniform without mutation. The obvious change of the intensity of the disturbance signal is the basis of identifying the disturbance signal by image processing. In order to further highlight the characteristics of the disturbance signal, image enhancement technology can be used to further highlight the disturbance part of the signal. The Gamma transform is a linear gray transformation. The formula is:

$$
y=(x+e s p)^{\gamma}
$$

The range of $\mathrm{X}$ and $\mathrm{Y}$ is [0,1]. $e s p$ is the compensation coefficient, and the $\gamma$ is Gamma coefficient? Gamma transform can selectively enhance contrast of low gray area or contrast of high gray area according to different values of $\gamma . \gamma$ is a very important parameter in image grayscale correction, and its value determines the gray level mapping between input images. Among them:

$\gamma>1$, the contrast of the high gray area of the image is enhanced;

$\gamma<1$, the low gray area contrast of the image is enhanced; $\gamma=1$, the grayscale transformation is linear, that is, the original image is not changed.

When performing Gamma transform, 0-255 of the gray scale dynamic range is usually converted to $0-1$, and then Gamma transform is used to restore the original range. After the Gamma transformation, the optimal threshold is selected by the method of the largest class variance. The gray level image is converted to two value image, which further highlights the distortion characteristics of the disturbance signal. The gray level of the grayscale image is $\mathrm{L}$, and the gray range is $[0, \mathrm{~L}-1]$. The best threshold formula for calculating the image using the maximum variance algorithm is as follows:

$$
\left.t=\operatorname{Max}\left[w 0(t) *(u 0(t)-u)^{2}+w 1(t) *(u 1(t)-u)^{2}\right)\right]
$$

Where the segmentation threshold is $t, w 0$ is the background ratio, $u 0$ is the background mean, $w 1$ is the foreground ratio, $u 1$ is the foreground mean, and $u$ is the mean of the whole image. The best threshold for segmenting image is the maximum value of $t$. The effect of Gamma transform on voltage sag signal is shown in Figure 2.

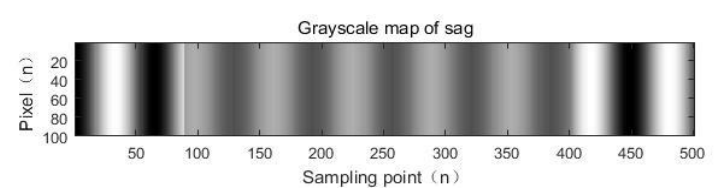

(b) 


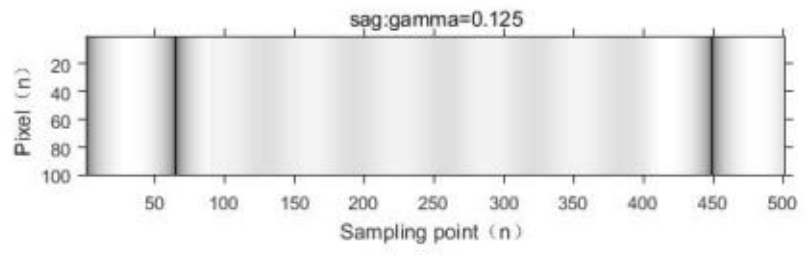

(c)

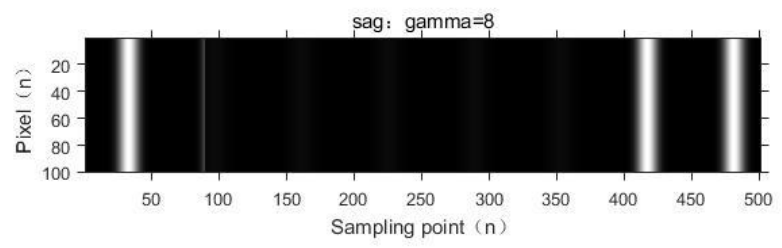

(e)

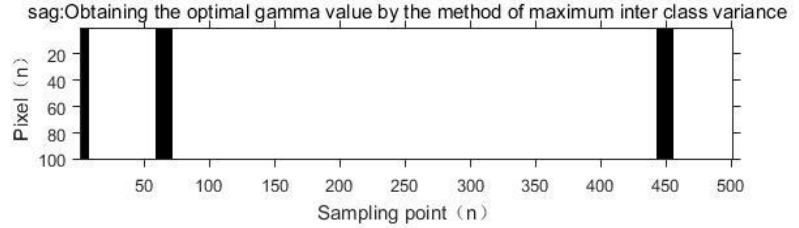

(d)

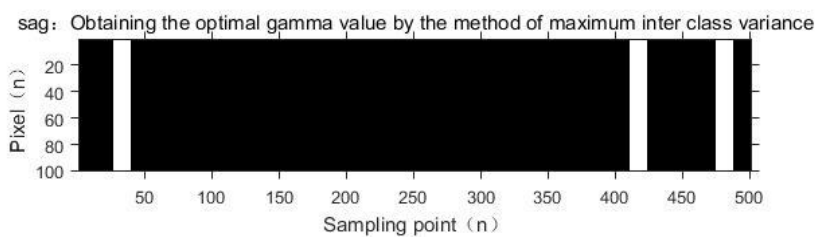

(f)

Figure 2: Effect Diagram of Sag Signal Processing by Gamma Transform

\section{IMAGE FEATURE EXTRACTION OF DISTURBANCE SIGNAL}

After obtaining the enhanced two value images, various typical image features can be extracted from the image to identify the disturbance signals. Here, the image area, the centroid and the Euler number are selected for the classification of the disturbance signals based on the features of the images based on the image. The centroid formula is as follows:

$$
r_{\sigma}=\frac{\sum_{i} m_{i} r_{i}}{M}
$$

$$
\text { Among them } M=\sum_{i=1}^{n}{ }_{i} \text {, }
$$

The Euler number A $(n, \mathrm{~K})$ means that all $n$ element permutations have exactly the number of $k-1$ ascending permutations.

\section{CLASSIFIER DESIGN}

There is a certain noise interference in the measured signal. At the same time, the distortion of power quality disturbance signal presents different characteristics and parameters changes with the specific factors such as disturbance source, load condition and power system structure. Therefore, the design of classifier needs to be carried out on the basis of a large number of statistical experiments to meet the needs of different application environments. The BP neural network is used to establish the feature classification model [7].

BP neural network is simple and clear, and has good applicability. It is one of the most widely used training algorithms of multi-level artificial neural network. The BP neural network is used to classify the features. The existing research has proved that the BP neural network containing single hidden layer can approximate any nonlinear function.

The training process of the BP neural network is based on the data of the training set, constantly adjusting the weights between the connected neurons, and finally establishing the process of the network model that meets the requirements. The training set of the BP nerve consists of the input vector and the ideal output vector. All the vectors in the training center should be the real results obtained through the simulation system, or the real data collected from the real system.

For the neuron, its input is:

$$
n e t=x_{1} w_{1}+x_{2} w_{2}+\cdots+x_{n} w_{n}
$$

Where $x_{1}, x_{2}, \cdots, x_{n}$ is the input of neurons, and $w_{1}, w_{2} \cdots w_{n}$ is the corresponding connection power. At the beginning of the training, a random number of smaller numbers is used to initialize the network weight to avoid the saturation state caused by the excessive initial value of the weight, and eventually lead to the failure of the training.

BP neural network needs to be trained one by one according to the training sample set, and BP neural network is continuously improved in training. Therefore, the outermost cycle of the BP neural network training algorithm is the requirement of the precision, followed by the training of the input and output of the training set sample. Therefore, if the BP neural network adjusts part of the weights in the course of training, even if the precision of the sample is not satisfied, it will no longer adjust it, and then continue to deal with other samples. When all samples in the set are adjusted, the above process can be repeated again. Finally, all the sample needs are met, and the network adjustment is over.

\section{EXPERIMENTAL SIMULATION RESULTS}

In order to show the effectiveness of the algorithm, firstly, electromagnetic transient simulation model is built to generate all kinds of power quality disturbance signals. Using Matlab to generate each disturbance signal, 500 groups are used to verify feature classification ability. The sampling rate of the signal is set to be $3.2 \mathrm{kHz}$, and the signal-to-noise ratio is $50 \mathrm{~dB}$. In order to figure out the correct statistical conclusion, the range of parameters can be covered as far as possible in the simulation process, such as different amplitude changes, disturbance duration and so on. Based on the simulation data samples obtained, the effect of feature combination classification is compared. In addition, the noise signal is generated by superimposed white noise are $40 \mathrm{~dB}, 30 \mathrm{~dB}$ and $20 \mathrm{~dB}$, respectively. And then each group of 100 sets is used to test the accuracy of classification. The final test results are shown in Table 1.

Table 1: Simulation results

\begin{tabular}{|lllll|}
\hline \multicolumn{5}{c}{ Classification accuracy (\%) } \\
Disturbance type & \multicolumn{4}{l}{} \\
\cline { 2 - 5 } & $50 \mathrm{~dB}$ & $40 \mathrm{~dB}$ & $30 \mathrm{~dB}$ & $20 \mathrm{~dB}$ \\
& & & & \\
\hline Sag & 100 & 99.2 & 96.8 & 88.4 \\
Swell & 100 & 99.8 & 96.9 & 86.1 \\
Harmonics & 100 & 100 & 93.8 & 85.6 \\
Interruption & 98.9 & 95.3 & 93.6 & 83.1 \\
Transient & 100 & 99.8 & 96.1 & 86.9 \\
Average accuracy & 99.78 & 98.82 & 95.44 & 86.02 \\
& & & & \\
\hline
\end{tabular}


Simulation results show that the proposed method can identify 5 typical power quality disturbances more accurately. It can be seen that the new method has higher recognition accuracy and has certain practical application value.

\section{ACKNOWLEDGMENT}

This work is partially supported by the Science and Technology Project of Jilin Province Education Department (Nos.JJKH20170219KJ); Science and Technology Program Project of Jilin Provincial Science and Technology Department (Nos.20180101336JC) and Science and Technology Innovation Development Plan Project of Jilin City(Nos. 201750239).

\section{REFERENCES}

[1] Saini, M.K., Kapoor, R. 2012. Classification of power quality events - A review [J]. Electrical Power and Energy Systems, 43, 11-19.

[2] Yin, B., Yigang, H.E., Zhu, Y. 2015. Detection and Classification of Power Quality Multi-Disturbances Based on Generalized S-transform and Fuzzy SOM Neural Network [J]. Proceedings of the Csee, 35, (4), 866-872.

[3] Gunal, S., Gerek, O.N., Ece, D.G., Edizkan, R. 2009. The search for optimal feature set in power quality event classification [J]. Expert Systems with Applications, 36, 10266-10273.

[4] Manimalaa, K., Selvi, K., Ahilaa, R. 2011. Hybrid soft computing techniques for feature selection and parameter optimization in power quality data mining [J]. Applied Soft Computing, 11, 5485-5497.

[5] Nantian, H., Dianguo, X., Xiaosheng, L., Lin, L. 2012. Power quality disturbances events recognition based on S-transform and probabilistic neural network [J]. Neurocomputing, 98 (S1), 12-23.

[6] Shareef, H., Mohamed, A., Ibrahim, A.A. 2013. An image processingbased method for power quality event identification [J]. Electrical Power and Energy Systems, (46), 184-197.

[7] Li, C., Xuan, W.C. 2017. Green Development Assessment of Smart City Based on PP-BP Intelligent Integrated and Future Prospect of Big Data. Acta Electronica Malaysia, 1 (1), 01-04. 